\title{
November 2017 Arizona Thoracic Society Notes
}

The November 2017 Arizona Thoracic Society meeting was held on Wednesday, November 15, 2017 at the HonorHealth Rehabilitation Hospital beginning at 6:30 PM. This was a dinner meeting with a lecture followed by case presentations. There were 15 in attendance representing the pulmonary, critical care, sleep, allergy, infectious disease and radiology communities.

At the beginning of the meeting several issues were discussed:

1. CME offered by the Southwest Journal of Pulmonary and Critical Care Medicine (SWJPCC) is currently offered to only the Southwest state thoracic societies and the Mayo Clinic. After discussion it was felt that this restriction of access was no longer appropriate and CME credits should be available to all.

2. Efforts continue to obtain CME for the Arizona Thoracic Society meetings. Our Chapter Representative, Dr. Gerry Schwartzberg, is approaching this with the American Thoracic Society. Locally, HonorHealth sent out a survey on CME needs. Members were encouraged to fill out the survey suggesting HonorHealth offer CME for these meetings.

3. No one was able to attend the last Tobacco 21 meeting in late October. In order to keep the Arizona Thoracic Society in the loop, Dr. Rick Robbins will contact the American Lung Association to clarify if any legislation has been proposed for this year.

4. Dr. Robbins also proposed conducting a survey of members through the SWJPCC rating various insurance plans.

"Eosinophils: A Potent Contributor to Disease in Severe Asthma" was presented by Kevin Murphy, MD from the University of Nebraska Medical Center in Omaha. Dr. Murphy reviewed the pathophysiology of eosinophils in asthma making use of a figure from Nat Immunol 2015;16:45-56. He also described the clinical usage of benralizumab made by AstraZeneca, the sponsor of the talk. Benralizumab induces apoptosis of eosinophils. On Tuesday, the FDA approved benralizumab for the add-on maintenance treatment of patients age 12 years and over with severe asthma with an eosinophilic phenotype. Questions included how an eosinophilic phenotype was defined and if the monoclonal antibody might work in asthma with a neutrophilic phenotype. Although data was lacking it was thought by Dr. Murphy that benralizumab would probably not be beneficial in a neutrophilic phenotype.

There were 4 case presentations:

1. Dr. Paul Conomos presented a middle-aged woman with long-standing rheumatoid arthritis who has been multiple therapies but none with the success of biologic therapies. She has a history of an atypical Mycobacterium in the skin in 2007 and on chest $\mathrm{x}$-ray she has progressive lung nodules. She 
was asymptomatic. Bronchoscopy was nondiagnostic and a needle biopsy of one of the nodules showed granuloma. Discussion on whether to proceed to video-assisted thorascopic surgery (VATS). The majority felt that continued observation was the most appropriate course at this time.

2. Dr. Lewis Wesselius presented a 52-year-old woman from lowa with a previous diagnosis of possible sarcoidosis. She complained of a cough and heaviness in her chest. Thoracic CT showed a left hilar mass. Bronchoscopy with endobronchial ultrasound was done in lowa but was nondiagnostic. A follow-up CT scan showed progression of the mass and that a left lung lesion had developed. Bronchoscopy was nondiagnostic. Histoplasmosis complement-fixing antibodies were positive at 1:16 (normal < 1:8). Needle biopsy of the mass showed chronic inflammation and VATS showed fibrosis with granulomatous inflammation. No cultures from any of the procedures were positive. She was placed on itraconazole and has clinically improved. Dr. Wesselius proposed that perhaps the patient has fibrosis with a variance of fibrosing mediastinitis (1).

3. Dr. Wesselius presented a 67-ear-old woman he had seen that afternoon with cough and sputum production who had nonpitting edema. Thoracic CT scan showed bronchiectasis but no pleural effusions. The patient's fingernails were discolored yellow typical of yellow nail syndrome.

4. Dr. Rick Robbins presented a 69-year-old man from Bismarck, ND who presented with cough and a rash typical erythema nodosum on the right ankle which had been present for 6 months. Chest $x$-ray showed multiple nodules in the right lung. Both coccidioidomycosis $\operatorname{lgM}$ and IgG were positive. The patient was begun on fluconazole and after a week felt better. In the context of Dr. Gerry Schwartzberg's recent Medical Image of the Week: Erythema Nodosum, Uncle Jun was right, "They come in threes." This case would be the third in addition to Dr. Schwartzberg's two cases.

There being no further business, the meeting was adjourned about 8:30 PM. The next meeting will be in Phoenix in January (date TBA) at 6:30 PM at HonorHealth Rehabilitation Hospital.

Richard A. Robbins, MD

\section{Reference}

1. Koksal D, Bayiz H, Mutluay N, Koyuncu A, Demirag F, Dagli G, Berktas B, Berkoglu M. Fibrosing mediastinitis mimicking bronchogenic carcinoma. $\mathrm{J}$ Thorac Dis. 2013 Feb;5(1):E5-7. [CrossRef] [PubMed] 